\title{
Cognitive Computing and its Relationship to Computing Methods and Advanced Computing from a Functional Modeling Perspective
}

\author{
Andy E. Williams, Nobeah Foundation, Nairobi, Kenya
}

\section{Keywords}

General Collective Intelligence, Artificial General Intelligence, cognitive computing, computing methods, advanced computing

\begin{abstract}
Recent advances in modeling human cognition have resulted in what is suggested to be the first model of Artificial General Intelligence (AGI) with the potential capacity for human-like general problem-solving ability, as well as a model for a General Collective Intelligence or GCI, which has been described as software that organizes a group into a single collective intelligence with the potential for vastly greater general problem-solving ability than any individual in the group. Both this model for GCI and this model for AGI require functional modeling of concepts that is complete in terms of meaning being self-contained in the model and not requiring interpretation based on information outside the model. This definition of a model for cognition has also been suggested to implicitly provide a semantic interpretation of functional models created within the functional modeling technique defined to meet the data format requirements of this AGI and GCI, so that the combination of the model of cognition to define an interpretation of meaning, and the functional modeling technique, together result in fully self-contained definitions of meaning that are suggested to be the first complete implementation of semantic modeling. With this semantic modeling, and with these models for AGI and GCI, cognitive computing is far better defined. This paper explores the various computing methods and advanced computing paradigms from the perspective of this cognitive computing.
\end{abstract}

\section{Introduction}

Cognitive computing is a fairly new field [1], [2], [3].This paper explores the implications of cognitive computing approaches (Artificial General Intelligence and General Collective Intelligence) on the development and execution of computing methods, where these cognitive computing approaches have been defined from a unique functional point of view. Though functionalism [4] has been well studied, and though the implications of functionalism on computability have also been explored [5], to the author's knowledge, no work other than that of the author has synthesized these concepts to define a model for cognitive computation from the functional point of view. Because this approach and its application are so new, there is no other work available to cite. So by necessity the examples discussed in this paper refer overwhelmingly to the author's own work. A platform containing a subset of AGI and GCI based computing currently being designed for a proposed large scale collective intelligence based program to accelerate achievement of the sustainable development goals gives cause for this exploration. Through defining functional models of computing infrastructure, where AGI and GCI are intended to enable those models to be used to develop software and hardware radically more quickly while enabling that development to benefit a vastly greater number of projects, this exploration aims to significantly accelerate the development of platforms in healthcare, education, and a wide variety of other areas, while also dramatically lowering costs. 
General Collective Intelligence or GCI has been described as software that organizes a group into a single collective intelligence with the potential for vastly greater general problemsolving ability than any individual in the group. A recently defined model of GCI incorporates a recently defined model of AGI [8] as intelligent agents to interact on behalf of users, to enable users to interact and achieve outcomes at potentially exponentially greater speed and scale [6], [7].

GCI is predicted to give groups the capacity to reliably define and solve problems that are "higher order" in that the problems are too complex to be defined and solutions too complex to be discovered by any individual human cognition, or by any human group without GCI [9]. Both this model for GCI and this model for AGI require functional modeling of concepts that is complete in terms of meaning being self-contained in the model and not requiring interpretation based on information outside the model. This definition of a model for cognition has also been suggested to implicitly provide a semantic interpretation of functional models created within the functional modeling technique defined to meet the data format requirements of this AGI and GCI, so that the combination of the model of cognition to define an interpretation of meaning, and the functional modeling technique, together result in fully self-contained definitions of meaning that are suggested to be the first complete implementation of semantic modeling [10].

\section{Why Consider AGI and GCI as a Basis for Navigating Computing Methods?}

Since potentially suitable models of AGI and GCI have just recently been defined, but have not yet been implemented, the question arises why an AGI and GCI based framework for navigating computing methods should be considered at this early stage, and how the highlevel properties of such a framework can be objectively validated and therefore add concrete value rather than being mere speculation. As for why consider such a framework, the simply answer is that doing so creates the potential to exponentially increase outcomes targeted by any computing methods, where doing so can be demonstrated to be outside the capacity of any human intellect to reliably achieve otherwise. As explored in the domain of sustainable housing design, any design process faces limits to the complexity it has the capacity to navigate [11]. Similarly, in the computing domain, where computing methods designed by any individual human without AGI, or by any group without GCI can only define the problems that can fit inside human cognition, and can only solve those problems with the solutions that are discoverable within human cognition, the combination of AGI and GCI can be demonstrated to have the capacity to reliably generate an exponential increase in general problem-solving ability [12]. Measured in impact on any outcome, such as the outcomes targeted through the design of computing methods, this can be expected to drive an exponential increase in impact on those outcomes.

AGI creates the potential to explore the fitness of every combination of different technologies in implementing every computing operation, and AGI creates the potential to explore interactions between computation operations that are much higher order (much more complex) than currently possible, and to do so at vastly greater speed and scale, in order to achieve vastly greater impact on any targeted outcomes of computation. GCI creates the potential to collectively store information about which combination of different technologies is most fit in implementing every computing operation, so that the computing operations executed by any one individual benefit from intelligence gained from the execution of any computing operation by any other individual. GCI also creates the potential to enable higher 
order interactions between computation operations that orchestrate group processes, and to do so at vastly greater speed and scale, in order to achieve vastly greater impact on targeted collective outcomes of computation. As for how the high-level properties of such a framework can be validated as being correct, that question is left for future exploration.

\section{Conceptual Example of the Use of Cognitive Computing to Navigate Computing Methods}

From the functional modeling perspective, cognitive systems consist of a well-defined set of functionality, including functional modeling. The model of cognitive computing discussed in this paper utilizes this set with the goal of achieving human-like computing (adaptive problem-solving in the space of concepts). One adaptive problem-solving process suggest to require human-like general problem-solving ability is generalizing problems in a way that makes it possible to see other better understood problems as equivalent to a poorly understood one, and to see solutions in a more abstract way that makes it possible to generalize solutions so they can be used to solve many other problems.

As a specific example, where the term "cognitive radio" describes a system that automatically adapts to use the best available band for communication, cognitive computing might address any problem of communication whatsoever. In a GCI orchestrated "Define Communication" process, intelligent agents based on some subset of AGI might work behalf of each user to negotiate the best available physical connection, electromagnetic spectrum, protocol at each protocol layer, network topology, and every other function of Internetworking and telecommunication. And GCI might orchestrate that cooperation to adaptively learn which implementation of each function is most fit in each context from all possible occurrences of Internet and telecommunication use. The usefulness of doing so would be expected to be increased sustainability across the entire product life-cycle, an increase in affordable access to communication, and an increase in quality of communication at each level of affordability. Because network changes or upgrades resulting in inoperable devices that have to be discarded would be expected to disappear. A conceptual example of why this sustainability is not reliably achievable without GCI has been explored in consumer electronics (headsets) [14]

And where operating systems have gained tremendous flexibility through essentially defining functional models of hardware through a Hardware Abstraction Layer (HAL), AGI based operating systems might increase that flexibility tremendously further still by defining functional models that abstract all functions of the operating system itself. Using GCI to orchestrate cooperation to adaptively learn from all possible uses by all individuals of any implementation of any operating system function, could enable an AGI to determine which implementation of a function is most fit in each context, whether that context be execution on a cell phone compatible with the Android OS, or a server compatible with some flavor of unix. When all instances of all operating systems then fall within a single functional model, in an AGI driven "Define Implementation of Operating System" process an intelligent agent might choose the best implementation on the fly. In other words, operating systems might construct themselves for any device in a way that enables any semanically defined software application to construct itself to run on it. And in addition to the design process, with the increased general problem-solving ability of GCI those intelligent agents might execute maintenance, and all other processes across the entire product life-cycle to self-assemble into structures with a level of complexity not achievable otherwise, and do so at speeds and scales 
that provide unbeatable competitive advantage for the group. But how might one systematically enumerate the opportunities to create such processes, and to implement them with all available computing methods so this is possible?

As described above, cognitive computing can be performed at the individual level with an intelligent agent consisting of some subset of AGI functionality, or at the group level with a platform consisting of some subset of GCI functionality. The "Define Communication" described above is a group process that might potentially be executed by a GCI, whether as an internal process of the GCI, or as an external tool. "Define Implementation of Operating System" is an individual process that might potentially be executed by an AGI, also as either an internal process, or as an external tool. This process might consume data on which implementation is more fit in a given context, where this information has been gathered by a group process, potentially one running within a GCI. Some types of problems can be addressed through the individual processing of an AGI are problems as involving the entire dataset distributed internally through the AGI (problems involving semantic level integration and human-like cognition), or problems involving dataset with centralized access that is accessible to the AGI (problems involving mass surveillance). Some types of problems that can be addressed only through group processing are problems where access to data is decentralized to each individual.

Generalization as a process of adaptive problem-solving is just one process requiring humanlike general problem-solving ability. How are the various computing methods relevant to execution of this and all other processes requiring such general problem-solving ability? Firstly, assume that an AGI or GCI exists. That AGI or GCI might execute an external software program that automates some processing operations. Or that AGI or GCI might gain an understanding of those processing operations and execute those operations internally. What types of problems can be addressed through processing internally within the AGI or GCI as opposed to externally? What is the benefit of processing internally within the AGI or GCI as opposed to externally?

Table 1: Benefits of executing computing within or outside a system of cognitive computation.

\section{Location of Positive and Negative Characteristics of Location \\ Process}

Internal to Pros: Process is "understood". Complete semantic level integration with other AGI/GCI cognitive processes enables creativity, generalization, and other characteristics of human-level general problem-solving ability to be applied to a process that is understood.

Cons: Processing can only occur at the speed of the cognitive system. In the functional model of cognition referred to in this paper this speed might be constrained by the boundaries of stability of the cognitive system. Resolution of concepts is limited by the resolution of the cognitive system.

External to Pros: Processing speed (operations per second) can potentially be increased AGI/GCI with the rate at which energy can be consumed, and processing scale (total number of operations) can potentially be increased to the scale at which materials are available to build the required hardware.

Cons: Process is not necessarily understood. 
Types of problems that are definable or solvable through executing computation within an AGI or GCI are expected to include problems and solutions requiring human-like cognitive attributes such as "adaptive problem-solving through generalization". Since cognitive computation in this functional model requires stability of operation, types of problems that are definable or solvable through executing computation outside an AGI or GCI include problems or solutions requiring computation greater than any limits of this stability. How are the various computing methods relevant in the case of individual or group processing? Or in the case of internal processing, or in the case of external processing?

Table 2: Relevance of computing methods for individual or group cognitive computing.

Model Requirements on Computational Processes Relevance of Computing Methods to be Executable within Generalization Based Adaptive Problem-Solving Process

AGI Semantic modeling. Complete semantic level For internal processing within an AGI, integration with cognitive system. computing methods must be invisible to the AGI if they are not to impact stability. For external processing outside of an AGI, computing methods can be used to determine fitness of the implementation of a given computing process.

GCI Semantic modeling that is also functional Unknown (subject of future modeling (an additional layer of abstraction investigation). required for groups to more easily exchange information). Complete semantic level integration with cognitive system.

\section{Background on the Cognitive Model}

In understanding how AGI and GCI must interact with all computing hardware or software, it is useful to understand the underlying model of cognition. In the model of individual and group human cognition discussed in this paper, the cognitive system moves through a space of concepts, or a "conceptual space". The path between two concepts is a "reasoning process" if directed, and an "understanding process" if passive. These spaces are spanned by a set of four minimal functions that are intended to have the capacity to compose any cognitive (reasoning or understanding) process. These paths or cognitive processes are represented by functional models with input concepts, output concepts, and additional concepts defining the context of execution. Similarly, since a problem in this functional modeling approach is the lack of such a path, functional models of problems have a similar representation.

Detection of where the cognitive system is in this conceptual space, and selection of the next cognitive process to execute, is performed by a "cognitive awareness" process. This model of cognition is part of a larger model of the entire human system that is defined by a Functional Modeling Framework (FMF) [15]. Similarly, in this larger model of the human system, the emotions operate within an emotional space, the body operates within a sensory space, and the consciousness operates within an awareness space. Where the model of the body has functional units providing the capacity to integrate signals from the distribution of sensory receptors into coherent sensations, the model of cognition has similar functional units to integrate signals from the distribution of neurons into concepts, and the consciousness has 
similar functional units to integrate perceptions from their distribution across the sensory, emotional, or cognitive space, in order to have the capacity for awareness of all the other systems (conscious self-awareness).

From this functional perspective the computing operations that occur inside a system of cognition based computing, whether AGI or GCI, are any operations executed within the conceptual state space of the cognitive system. For computing operations to be executed within the conceptual state space of the system they must be executable by the system (i.e. by the cognitive awareness process), and those operations must provide the cognitive system with access to semantic models of all inputs, outputs, and context so that their execution can potentially be integrated with the execution of any other process within that system of cognition. This integration is described here as "semantic level integration" for convenience.

By contrast, external computing operations (such as computing the result of an arithmetic equation) are executed not by the cognitive awareness process, but by a reasoning process executed by the cognitive awareness process (reasoning through the process of interacting with the computer doing the calculation). External computing operations (such as those required to compute the result) might be modeled semantically, and execution of those operations might be initiated by the system of cognition, but where the system of cognition lacks access to interact with a semantic model of the intermediate processing, that processing cannot be integrated with other processing in that system of cognition. Because integration of that computational tool with human-like processes such as creativity is impossible, creativity might be possible in reasoning processes through which the cognitive system might adapt its use of the tool, but not possible in directly adapting the tool itself, except through the interfaces the tool provides for that adaptation.

\section{Understanding the Interaction of a Cognitive Computing System with Hardware and Software by Analogy}

Hardware and software then consists of external computing hardware or software that can be considered to be tools of the cognitive computing system, and computing hardware and software that can be considered to be part of the cognitive computing system. All connected hardware that is semantically integrated becomes part of the individual "body" for an AGI and part of the collective "body" for a GCI. Similarly, all processing that is integrated at the semantic level becomes reasoning processes that can be executed within the AGI, or collective reasoning processes that can be executed within the collective cognition. All processing that is not semantically integrated becomes a tool that can be executed. Exactly what hardware and software processes must be integrated at a semantic level with any system of cognitive computing, and what the criteria for "semantic level integration" are, is the subject of ongoing investigation.

The analogy with the human organism has limits. In a body there are specific structures with a specific function. In a system of cognitive computing, functional components are abstractions, with a potentially great many implementations. In the case of network connectivity, a GCI might select whatever implementation of a given function that is most fit at achieving the targeted outcome in a given context. Where an individual human requires their spinal column to be functioning in order to live, a GCI might not need any particular instance of hardware infrastructure for connectivity, but instead might simply need some hardware infrastructure to 
provide that function. The capacity to manipulate tools at this higher level of abstraction places specific requirements on user interfaces and other computing functionality.

As an example of abstraction, the GCI based "Define Communication' process mentioned earlier might involve simultaneous interaction with all devices in the GCI to define the optimal local network topology in each residential neighborhood, then the optimal network topology in each section of the city, and so forth until the optimal global network was defined. These multiple hierarchical layers of interaction might result in networks self-assembling into an interaction of higher order complexity than an individual without GCI could design. As another example, designing computing hardware in terms of such functional models permits design processes in which the GCI varies the functionality in each functional component, as well as varying the interfaces to that functionality, so that the collective can explore all possible design configurations and reliably adapt to utilize the best design components created by any individuals in the group. This is intended to replicate the capacity of nature's evolutionary design process to navigate a vast potential design configuration space, creating the potential to self-assemble structures with levels of complexity not possible today. Current hardware and software designs can only solve problems their designers can understand, and can only do so with solutions their designers are able to discover. Of course, using such a process would be expected to require vastly greater general problem-solving ability to navigate the resulting complexity. If GCI does indeed have the potential to drive an exponential increase in general problem-solving ability, applying that ability to such a process to design computational methods that have never before in human history been possible, as well as applying that ability to collective problems in general, has been suggested to potentially be the most important impact in human history, and the most important impact in the immediate future of human civilization until the transition to a second order GCI creates the potential for another exponential increase [13].

\section{Application in Navigating Computing Methods and Advanced Computing}

One functional model of computing is provided by a proposed eXtensible Domain Modeling Framework (XDMF) [16] that attempts to define minimal functional models of processes in each domain, that can be used to compose all other processes in that domain, so that those models might be represented using Domain Specific Modeling (DSM) tools, and so those functions might be implemented, and models automatically translated into implementations by those DSM tools. Along with defining domains that attempt to represent all business process operations across the entire product and service life-cycle from marketing, and sales, to service, and recycling, the XDMF attempts to represent domains such as the UI (containing user interface operations), and the Information Processing Domain (containing information processing operations). Consider the case of one of these IPD operations, the LOOKUP operation.

Table 3: Use of computing methods to categorize fitness of implementations.
IPD
Computing Methods Involved in
Specific Context of Usage (Fitness of
Operation
LOOKUP
Specific Implementation
Implementation in that Context)
LOOKUP
Secure Computing, Cloud Computing Insecure Hosted Environment (High Fitness)
Secure Computing, Cloud Computing Disconnected, Performance Critical Personal Environment (Poor Fitness) 
In this way, in cognitive computing, such as AGI and GCI based computing paradigm, terms like: pervasive computing, green computing, grid computing, soft computing, cloud computing, cyber security, and the other computing and advanced computing methods mentioned in the call for papers for these proceedings refer to categories that might be used to classify implementations according to their fitness in different contexts. All these computing methods might be semantically integrated, and therefore occur within the boundaries of an AGI or GCI. But given that the semantic modeling involved might require human-like general problem-solving ability, which without AGI must be done manually, it may be some time before semantic integration of computing methods can be achieved. In the mean time, all nonsemantically integrated computing, and all the computing methods such computing contains, might be implemented in a great many different ways depending on the context. This classification combined with the use of even a subset of GCI has the potential to significantly increase collective capacity to navigate these computing methods and their implementations.

\section{Directions Forward}

In order to define the complete AGI and GCI based computing paradigm, much work is still required to define functional models of both hardware computing processes, as well as software computing processes that might occur in either applications or operating systems. However, in some domains, such as the User Interface Domain, or the Information Processing Domain, functional models, as well as reference implementations of each functional component in those functional models, have already been proposed. With this work, practical examples implementing a subset of this functionality are currently being developed such as the platform containing a subset of AGI and GCI based computing currently being designed for a proposed large scale collective intelligence based program to accelerate achievement of the sustainable development goals. Many research questions remain. For one, the implications of defining what is within or outside of a cognitive computing based system in terms of semantic integration must be explored. For example, hardware or software might be semantically integrated with multiple cognitive computing system instances, switching between them when required, and therefore might be "within" several instances of such cognitive systems.

\section{Conclusion}

All computing methods represent cognitive processes. If these processes are individual and outside the boundaries of an AGI they represent automation of individual reasoning or understanding processes. And if these processes are collective and outside the boundaries of a GCI they represent automation of collective reasoning or understanding processes. Both are implemented by external computing methods. Cognitive computing is a brave new world in which semantic modeling of hardware and software has the potential to introduce the possibility of another level of abstraction for developers. With this abstraction, computing methods might be elaborated and potential applications for those methods might be found at exponentially greater speed and scale. And developers of computing methods might gain the capacity to cooperate across an unlimited number of such methods to achieve levels of integration, and complexity of functionality that is not possible today. Where processes like "Define Communication" aim to increase impact on problems through being executed as tools by the adaptive problem-solving of cognitive computing, by generalizing models of these processes to be more common, and by classifying them according to computing methods, it may be possible to not only find other opportunities to increase impact this way, but to systematically find them. 


\section{References}

[1] (2015). Emerging Cognitive Computing Areas. In Cognitive Computing and Big Data Analytics (eds J. Hurwitz, M. Kaufman and A. Bowles). doi:10.1002/9781119183648.ch13 [2] Shaofei Wu, Mingqing Wang, Yuntao Zou, Bidirectional cognitive computing method supported by cloud technology, Cognitive Systems Research, Volume 52, 2018, Pages 615621, ISSN 1389-0417, https://doi.org/10.1016/j.cogsys.2018.07.035.

[3] Wang, Y., Baciu, G., Yao, Y., Kinsner, W., Chan, K., Zhang, B., Hameroff, S., Zhong, N., Hunag, C., Goertzel, B., Miao, D., Sugawara, K., Wang, G., You, J., Zhang, D., \& Zhu, H. (2010). Perspectives on Cognitive Informatics and Cognitive Computing. International Journal of Cognitive Informatics and Natural Intelligence (IJCINI), 4(1), 1-29. doi:10.4018/jcini.2010010101

[4] Boucher, S.C. Functionalism and structuralism as philosophical stances: van Fraassen meets the philosophy of biology. Biol Philos 30, 383-403 (2015).

https://doi.org/10.1007/s10539-014-9453-z

[5] Ned J. Block, Functionalism, Editor(s): L. Jonathan Cohen, Jerzy Łoś, Helmut Pfeiffer, Klaus-Peter Podewski, Studies in Logic and the Foundations of Mathematics, Elsevier, Volume 104, 1982, Pages 519-539, ISSN 0049-237X, ISBN 9780444854230, https://doi.org/ 10.1016/S0049-237X(09)70217-4.

[6] Williams, A. E. (2020, April 30). A Model for General Collective Intelligence. https://doi.org/10.31730/osf.io/6u984

[7] The Relationship Between Collective Intelligence and One Model of General Collective Intelligence, Andy E. Williams, Computational Collective Intelligence, 11th International Conference, ICCCI 2019, Hendaye, France, September 4-6, 2019, Proceedings, Part II, Pages 589-600

[8] Andy E. Williams, A Model for Artificial General Intelligence, Chapter, Artificial General Intelligence, Series: Lecture Notes in Computer Science, Year: 2020, Volume 12177, Pages 357-369, DOI: 10.1007/978-3-030-52152-3_38

[9] Definition of Higher Order Problems

[10] Andy E. Williams, Using Human-Centric Functional Modeling and a Functional Model of Cognition to Define a Complete Model of Semantic Meaning, working paper (2020)

[11] Limits to Design Cognition

[12] Williams, A. E. (2020, July 11). Human Intelligence and General Collective Intelligence as Phase Changes in Animal Intelligence. https://doi.org/10.31234/osf.io/dr8qn

[13] Williams, A. E. (2020, August 25). Assessing the Potential Impact of General Collective Intelligence. https://doi.org/10.31730/osf.io/e5usx

[14] Andy E. Williams, General Collective Intelligence as the Emerging Paradigm in Design for Sustainable Development, in print, accepted to the proceedings of ISDRS 2020

[15] Williams, A. E. (2020, April 16). A Human-Centric Functional Modeling Framework for Defining and Comparing Models of Consciousness and Cognition.

https://doi.org/10.31234/osf.io/94gw3

[16] Andy E. Williams, eXtensible Domain Modeling Framework, working paper (2009) 